\title{
Glaucoma in Fuchs' heterochromic cyclitis associated with congenital Horner's syndrome
}

\author{
LUCIAN S REGENBOGEN AND NAVA NAVEH-FLOMAN \\ From the Maurice and Gabriela Goldschlager Eye Institute, Sheba Medical Center, Tel Hashomer, Sackler \\ School of Medicine, Tel Aviv University, Tel Aviv, Israel
}

SUMMARY We report a retrospective study of five patients with monocular Fuchs' heterochromic cyclitis associated with an ipsilateral Horner's syndrome. The minimum follow-up was 10 years. The presenting findings were cyclitis in three of the patients and heterochromia iridis associated with blepharoptosis in the other two. The major factors affecting all five patients were cataract and glaucoma. The intraocular pressure was uncontrolled even with maximal therapy, and antiglaucomatous surgery was performed in all cases. A short period of good postoperative control was followed by an intractable ocular hypertension, causing loss of useful vision in all patients. The remarkable combination of Horner's syndrome with glaucoma and their interaction is discussed.

Fuchs' heterochromic cyclitis (FHC) is a commoner ocular disorder than has generally been recognised. ${ }^{-9}$ It is frequently overlooked when the heterochromia is slight or when all its clinical features are not present at the same time.

One of the most serious complications of the disease is glaucoma, which has been observed in $18.6 \%$ of the studied cases. ${ }^{6}$ Although there is no unanimity on the prognosis for the glaucoma in this syndrome,$^{357}$ recent communications ${ }^{810}$ suggest that it usually becomes resistant to medical therapy, and surgery is only moderately successful.

The purpose of this report is to describe the manifestations of unilateral FHC associated with Horner's syndrome and complicated by ipsilateral glaucoma in five patients. To the best of our knowledge, no similar cases have been previously reported. We discuss the significance of the association and the implications regarding the unfavourable course of the glaucoma in these patients.

\section{Case reports}

\section{CASE 1}

A 53-year-old male was first seen in May 1970 because of a progressive decrease in visual acuity, elevated intraocular pressure (IOP), and recurrent

Correspondence to Professor L S Regenbogen, Maurice and Gabriela Goldschlager Eye Institute, Sheba Medical Center, Tel Hashomer 52621, Israel. inflammations in the left eye during the preceding three years. Drooping of the eyelid and discoloration of the iris had been evident in the affected eye since early childhood.

His best corrected visual acuity was $6 / 7(20 / 25)$ in the right eye (RE) and limited to light perception in the left eye (LE). The intraocular pressure (IOP) in the LE while the patient was on $4 \%$ pilocarpine and acetazolamide $250 \mathrm{mg}$ was $28 \mathrm{mmHg}$. The IOP in the RE was $14 \mathrm{mmHg}$, and when the patient was submitted to a provocative topical dexamethasone test the eye did not react with a significant rise in tension.

External examination of the LE revealed $2 \mathrm{~mm}$ blepharoptosis. Slit-lamp biomicroscopy disclosed a normal RE and a few white keratic precipitates (KP) with flare and cells in the anterior chamber in the LE. The right iris was brown and rich in crypts in marked contrast to that of the left eye, which was light grey, without crypts, and showed transillumination throughout an extensive depigmented area. Gonioscopy showed a few engorged blood vessels in the LE and wide open angles in both eyes. Ophthalmoscopic examination showed a normal right fundus, whereas visualisation in the LE was impossible owing to an almost mature cataract.

Two weeks after discontinuation of topical antiglaucoma medication both pupils reacted to light and accommodation, and miosis of $1.5 \mathrm{~mm}$ was disclosed in the LE; $4 \%$ cocaine hydrochloride failed to dilate the left pupil but fully dilated the right. Marked left 
pupillary dilatation was observed after further instillations of $1 \%$ epinephrine.

In January 1972 cyclodialysis was performed in the LE because of uncontrollable IOP despite supplementary medication. After an apparently good postoperative period of three months the IOP increased progressively to $30 \mathrm{mmHg}$ despite resumption of medical therapy.

After an uneventful left intracapsular extraction carried out in April 1972, which restored the vision to $6 / 10$, a 0.40 glaucomatous cupping and involvement of the inferior temporal rim were noticed. The left visual field showed a small step. In March 1977 trabeculectomy was performed because of further deterioration in the left visual field. A $2 \mathrm{~mm}$ hyphaema was noted after the operation. Transient good postoperative control of the IOP was followed by persistent elevation to $35 \mathrm{mmHg}$. The patient was not seen again until 1984, when the IOP in the LE was uncontrolled despite maximum medical therapy. The optic disc was totally cupped, and the visual field was reduced to 'tubular', with an additional isolated temporal island. The RE remained normal.

CASE 2

A 51-year-old female was referred for consultation in January 1973 because of a history of recurrent inflammation and decreased vision in the LE. During the previous 10 months the affected eye had developed ocular hypertension, and $4 \%$ pilocarpine and prednisone were administered.

On examination her corrected visual acuity was RE 6/6 (20/20) and LE counting fingers. The IOP was $25 \mathrm{mmHg}$ in the LE (under topical medication) and $15 \mathrm{mmHg}$ in the RE. The RE was normal, and its IOP did not react to topical steroid testing.

The left upper eyelid had $2 \mathrm{~mm}$ of blepharoptosis with good elevator function; a picture taken when the patient was 10 years old confirmed the presence of this abnormality since childhood. The right eyelid was normal.

Biomicroscopy disclosed typical FHC findings with a mature cataract in the LE. Gonioscopic examination revealed open angles with ciliary processes visible. A few large vessels were seen in the angle of the LE.

The left pupil was miotic and unresponsive to $4 \%$ cocaine (administered while the patient was off pilocarpine treatment for 10 days), but dilatation was observed after repeated instillations of $1 \%$ epinephrine. The right pupil reacted normally.

Uneventful left intracapsular cataract extraction was performed in January 1974 with subsequent improvement in visual acuity to $6 / 12(20 / 40)$. At this stage both optic discs were normal.

During the following years the IOP in the LE remained uncontrolled $(32 \mathrm{mmHg})$ despite maximum medical therapy. The left optic disc became progressively damaged, and the visual field showed a significant superior nasal scotoma. In July 1978 trabeculectomy was performed, with subsequent bleeding into the anterior chamber. The hyphaema, not absorbed after seven postoperative days, required surgical removal. No filtering bleb developed, though the IOP decreased to $15 \mathrm{mmHg}$.

The patient was seen incidentally in April 1983 because of a traumatic corneal ulcer in the RE. The IOP was RE $14 \mathrm{mmHg}$ and LE $30 \mathrm{mmHg}$ despite maximally tolerated medical therapy. In the LE vision was reduced to $1 / 20$, and the visual field consisted of a central zone connected by a narrow bridge to a temporal island due to a totally cupped optic disc.

\section{CASE 3}

A 60-year-old female presented in March 1975 with a 48-hour history of loss of vision in her painful RE. The patient claimed to have had a 'smaller' right eye and ipsilateral iris hypochromia since childhood. For many years she had received intermittent topical corticosteroids for chronic inflammation in the RE.

Ocular examination revealed visual acuity limited to light perception in the RE and 6/6 (20/20) in the LE. The anterior segment of the RE showed blepharoptosis of $2.5 \mathrm{~mm}$, with the lower lid slightly higher than that of the left side. Biomicroscopy of the RE disclosed perikeratic congestion, hazy cornea, a few hyaline posterior deposits, turbid aqueous, a hypochromic iris, and a hypermature cataract. The LE was normal. Applanation tonometry values were RE $50 \mathrm{mmHg}$ and LE $18 \mathrm{mmHg}$. On gonioscopy the angles were open. Right phakolytic glaucoma was diagnosed, and uneventful intracapsular cataract extraction was performed. After the operation the best corrected visual acuity in the RE improved to $6 / 9$ (20/30), and the IOP dropped to $22 \mathrm{mmHg}$. The right optic disc had a deep cup with involvement of the inferotemporal rim and a corresponding visual field defect. The iridocorneal angle remained open all around without synechiae. A few months later the IOP progressively increased to $30 \mathrm{mmHg}$ and remained at this level despite maximal medical therapy.

Cyclodialysis was performed in February 1976, but its hypotensive effect was only transitory. Six months later the IOP increased again to $32 \mathrm{mmHg}$ despite maximal topical and systemic medication. In the 10 years' follow-up the IOP remained uncontrolled and the optic disc was progressively damaged. In the unaffected eye the IOP remained normal, and a dexamethasone test was negative.

The patient refused additional surgical treatment. 
CASE 4

A 52-year-old woman was first seen in December 1971 because of a 14-year history of heterochromic cyclitis, cataract, and ocular hypertension in her RE. The ocular inflammation and the IOP responded favourably to topical therapy. The patient reported that the affected eye had been 'smaller' than the left eye ever since she could remember.

Slight enophthalmos and blepharoptosis of $2 \mathrm{~mm}$ with dropping of the lower lids was noted in the affected eye.

Best corrected visual acuity was RE 6/60 (20/200) and LE 6/7.5 (20/25). The IOP in the RE was controlled with $4 \%$ pilocarpine and acetazolamide $250 \mathrm{mg}$. The IOP in the LE was normal and did not react to topical corticosteroid testing.

Biomicroscopy of the RE disclosed a few dispersed KP and minimal flare in the anterior chamber. The iris was hypochromic, with a dull surface, and showing patchy areas of transillumination. Gonioscopically the angle was partially closed in the upper half but otherwise open to the ciliary body. A posterior subcapsular immature cataract was present. The optic disc seemed normal. The LE was absolutely normal.

The miotic right pupil, two weeks after discontinuation of topical medication, reacted normally to light and accommodation but failed to respond to a $4 \%$ cocaine test, whereas the normal opposite pupil dilated widely.

In June 1973 an uneventful extracapsular extraction in the RE followed by discission of a secondary cataract restored vision to $6 / 18(20 / 60)$. After surgery slight atrophy of the optic disc was disclosed, and a nasal field defect was detected. The IOP was normal but after 10 months measured $30 \mathrm{mmHg}$ despite. maximally tolerated medical therapy. Cyclodialysis performed in April 1974 controlled the IOP for a period of eight months, but was followed by refractory ocular hypertension during the subsequent years. A Scheie filtering procedure performed in March 1977 was followed by hyphaema and had a hypotensive effect that lasted only a year after surgery.

In December 1984 a follow-up examination showed that the right optic disc was totally damaged, and the visual field was reduced to two temporal islands. The LE remained normal.

\section{CASE 5}

A 56-year-old male was referred to us in May 1974 because of a gradual decrease in visual acuity in the LE during the preceding three years. The patient had a long history of repeated episodes of inflammation, as well as a lighter coloured iris in that eye. He also reported that the left upper lid had drooped since his youth.
On ocular examination his best refracted visual acuity was RE 6/6 (20/20) and LE 6/24 (20/80). External examination of the LE revealed a blepharoptosis of $2.5 \mathrm{~mm}$; slit-lamp biomicroscopy disclosed in the LE a few KP with filaments and a hypochromic iris. The normal crisp appearance of the iris was absent, and the iris surface was blurred. A transillumination defect of the iris and gaps in the pigment ruff were noted. Gonioscopy revealed an open angle with few dilated blood vessels. The lens showed an incipient subcapsular posterior cataract. A deep cup was noted. The IOP was $30 \mathrm{mmHg}$, and a small nasal defect was disclosed in the visual field.

The RE was completely normal. Its IOP was 12 $\mathrm{mmHg}$, and the eye did not react to a provocative topical dexamethasone test.

Both pupils reacted normally to light and accommodation. Miosis of $1.5 \mathrm{~mm}$ was present in the LE. After instillation of $4 \%$ cocaine the left pupil remained unchanged, while the right pupil reacted with $5 \mathrm{~mm}$ dilatation.

Over a one-year period the IOP in the LE remained uncontrolled despite strong topical and systemic medication, and a significant progression in loss of visual field was disclosed. Trabeculectomy was performed in January 1976. Hyphaema occurred during surgery. No distinct conjunctival bleb developed postoperatively. The IOP remained controlled for nine months without medication. In May 1977 extensive rubeosis was noted, and the IOP increased to $36 \mathrm{mmHg}$ despite the reinstitution of antiglaucoma medical therapy. The patient was lost to follow-up, but when he was last seen, in May 1984, the IOP in the LE exceeded $40 \mathrm{mmHg}$, and vision was reduced to temporal light perception only. The left optic disc was totally cupped. The RE remained normal.

\section{Discussion}

The distinctive features of FHC are slowly progressive uveitis with non-pigmented KP, minimal cells and flares in the anterior chamber, atrophy of the iris with hypochromia, and absence of posterior synechiae. The disorder is often associated with cataract and sometimes with glaucoma.

The aetiology of FHC is still subject to controversy. Some investigators consider that we are dealing with an immunological disease related to a defect in supressor T-cell activity. ${ }^{10}$ Earlier theories pointed to the possible role of an initial infectious process. ${ }^{1}$ More recent studies have demonstrated chorioretinal lesions, particularly suggestive of toxoplasmosis. ${ }^{112}$ The neurogenic theory of Passow ${ }^{13}$ proposed that damage to the sympathetic nervous system can mediate the changes in $\mathrm{FHC}$; the pre- 
sumption originates from the fact that sympathetic lesions due to neonatal injury may be followed by ipsilateral iris hypochromia. The hereditary theory ${ }^{6}$ originates from the fact that the more important types of iris heterochromia are hereditary in origin.

Horner's syndrome is an uncommon condition deriving from an interruption of the sympathetic pathway at any point along its course between the hypothalamus and the orbit. The syndrome accompanied by iris heterochromia usually indicates a longstanding cervical sympathetic denervation.

We report here a follow-up for 10 to 14 years of five patients with monocular FHC associated with ipsilateral Horner's syndrome and complicated by glaucoma. The diagnosis of FHC was based on the presence of the above mentioned features of the condition. The diagnosis of Horner's syndrome was based on the presence of blepharoptosis and miosis and the failure of the affected pupil to respond to $4 \%$ cocaine, whereas the opposite pupil dilated briskly. In every case Horner's syndrome was considered to be of congenital origin, as a 'smaller' eye had been noted since early childhood.

Cyclitis was the presenting sign of FHC in three patients (cases 2, 4, and 5) followed by iris hypochromia. In the two other patients (cases 1 and 3) heterochromia associated with blepharoptosis was the first apparent finding of the syndrome, occurring during youth and preceding other signs of uveitis by 20 to 30 years. In this regard $\mathrm{O}^{\prime}$ Connor ${ }^{10}$ confirmed that a significant number of patients with FHC have iris heterochromia since birth or early childhood.

We believe that the detection of a previous ipsilateral Horner's syndrome, as in cases 1 and 3, suggests the possibility that sympathetic damage exerts a trophic influence on the development of iris hypochromia and perhaps mediates other changes in FHC. In cases 2,4 , and 5 both immunological and/or sympathetic mechanisms may be taken into consideration. In this connection Calmettes and associates $^{14}$ reported a remarkable case in which Horner's syndrome following stellate ganglionectomy and FHC developed consecutively in the same eye.

Cataract was a major problem affecting all five patients in the current series: four patients developed posterior subcapsular cataract within four to 20 years after the first manifestation of FHC; in one patient (case 5) cataract was detected very early in the course of the disease. Four patients underwent uneventful cataract extraction (three intracapsular and one extracapsular) with prompt improvement in vision. In our experience the prognosis for cataract extraction in FHC is fair and the operation is no more likely to be complicated than any routine cataract surgery. The cyclitis was not worse after than before the operation. This confirms the good prognosis noted by
Franceschetti ${ }^{2}$ and Smith and O'Connor ${ }^{15}$ but differs from the experience of Ward and Hart, ${ }^{16}$ Norn, ${ }^{17}$ and Liesegang, ${ }^{8}$ who found a high prevalence of hyphaema, extensive peripheral synechiae, corneal oedema, and persistent pupillary membranes. Our results also differ from the findings of Hart and Ward ${ }^{18}$ which indicated that lens extraction has a deleterious effect on the dynamics of the IOP in eyes with FHC. Because previous patients with FHC who underwent extracapsular cataract extraction with posterior chamber lens implantation did not show a tendency to ocular hypertension, ${ }^{11}$ the use of this procedure seems advisable, especially in monocular cases.

The most serious complication in FHC remains the development of glaucoma. There has been a statistically significant increase in the incidence of this complication over the last 30 years. Whether the increase is merely an expression of improved diagnostic methods and better attention or is due to indiscriminate employment of topical steroids remains uncertain.

The cause of glaucoma in FHC is poorly understood. Huber ${ }^{3}$ considered the main cause of ocular hypertension to be an increase of the outflow resistance located in the trabeculum. Chandler and Grant ${ }^{19}$ stated that rubeosis of the iris in the anterior chamber angle induces the development of chronic secondary glaucoma.

In the current series all five patients with unilateral FHC had ipsilateral ocular hypertension resembling chronic open angle glaucoma in its course and symptomatology. One patient (case 3) presented with phakolytic glaucoma at the time of initial diagnosis, but after the cataract extraction extensive damage to the optic disc indicated also long-standing open angle glaucoma. The onset and duration of glaucoma varied: in one of the patients (case 4) glaucoma became evident concurrently with the appearance of cyclitis, while it was only a subsequent development in the remaining four patients. All patients developed glaucoma before cataract extraction.

There is no general agreement on the prognosis of glaucoma in FHC, and most authors report their conclusions on cases with a short control period. Some workers ${ }^{721}$ claimed a favourable outlook when the glaucoma was treated early. Other studies ${ }^{410}$ suggest that glaucoma usually becomes refractory to medical treatment. In the series reported by Liesegang, ${ }^{8}$ the prognosis was poorer than previously reported, although the author demonstrated success in controlling IOP by combined medical and surgical therapy in 21 of 32 patients.

In the current series maximum medical therapy (two topical agents with or without a carbonic 
anhydrase inhibitor) and cataract extraction were unsuccessful in controlling the IOP. Therefore antiglaucomatous surgery was performed in all cases: three patients (cases 1,2, and 5) underwent trabeculectomy, one patient (case 3) cyclodialysis, and one patient (case 4) cyclodialysis followed by a Scheie filtering operation. None of these procedures, though performed correctly, presented a well formed functioning conjunctival bleb. Hyphaema was frequent, and immediate postoperative complications and extensive rubeosis developed in one patient (case 5) a few months after glaucoma surgery. In all cases surgical procedures resulted in a short-term hypotensive effect followed by intractable ocular hypertension. In some of these patients the vascular accidents appear to have compromised the surgical result.

The cases in our study were selected out of a large group of 56 patients with FHC who were under long-term observation at the same period. Eight additional cases were also complicated by glaucoma but were not associated with Horner's syndrome. Of these cases (two of them in the aphakic eye), the IOP could be kept under control with medical treatment in only one patient. Though the number of cases is small and statistically insignificant, it appears that in more than $90 \%$ of patients the glaucoma, if the follow-up period is prolonged, becomes completely refractory to any form of therapy and is eventually the cause of loss of useful vision. In this matter our experience is different from all statistics showing a better prognosis.

No IOP abnormalities were detected in the uninvolved eye of patients in the present series. A provocative topical dexamethasone test was carried out in these eyes; only one fellow eye reacted with a significant rise in IOP and was classified as an intermediate steroid responder.

The remarkable combination of Horner's syndrome with glaucoma and their interaction seems inconsistent with what had been expected in such cases. The relationship between Horner's syndrome and IOP has been studied both in animal experiments and by clinical observations in patients who have suffered sympathetic denervation. Experimental studies, mainly in rabbits ${ }^{21}$ and cats, ${ }^{22}$ produced a short-term reduction of IOP following sympathectomy; long-term effects appear to have been variable or absent.

Cervical sympathectomy, by excision of the superior cervical ganglion, was used for the treatment of chronic simple glaucoma at the turn of the century. ${ }^{2324}$ Swegmark ${ }^{25}$ studied aqueous dynamics in patients with unilateral Horner's syndrome and concluded that sympathetic denervation reduced aqueous production and thus IOP in the ipsilateral eye. Wentworth and Brubanker ${ }^{26}$ found a statistically significant reduction of IOP in 21 patients with third nerve Horner's syndrome.

Because of the rarity of the clinical reports associating Horner's syndrome and glaucoma in the same eye, it is difficult to speculate on their relation. However, Julien and Vincendeau ${ }^{5}$ reported a case of FHC complicated by glaucoma that showed homolateral sympathetic injury after high dosage of amphetamine ingestion. Weinstein and Laugham ${ }^{27}$ described a patient with congenital Horner's syndrome in whom bilateral open glaucoma was detected at the age of 57 years. Brazier ${ }^{28}$ reported a case of bilateral cervical sympathectomy associated with unilateral Horner's syndrome in which the IOP was normal in the eye with Horner's syndrome, but ocular hypertension (in the presence of normal visual field and optic disc) was disclosed in the contralateral eye. The author suggested that the normal IOP in the eye with Horner's syndrome, and the normal visual field and optic disc in the fellow eye, might be due to some antiglaucomatous 'protective' mechanism produced by the sympathetic denervation. In view of the hypotensive effects of sympathetic lesions on ocular dynamic ${ }^{2326}$ one is surprised that, in cases with FHC, the associated Horner's syndrome in the ipsilateral eye had no prophylactic, or at least, alleviating influence on the complicating glaucoma.

In the cases described here the mechanism of the interaction between Horner's syndrome and glaucoma may have several possible causes. First, the occurrence of these two conditions was entirely independent and simply coincidental. A second possibility might be that Horner's syndrome is, if not responsible for the glaucoma, then at least an aggravating factor. This is unlikely, because there is no clinical or experimental evidence that the IOP can rise as a result of ocular sympathetic denervation in humans. A third possibility is that Horner's syndrome preceded the development of the cyclitis and glaucoma by many years and might have been associated with normal or hypotensive IOP before and in the first stages of the FHC; later, with the aggravation of cyclitis, the augmented protein levels in the anterior chamber produced inflammatory damage to the trabeculum, overcoming the eventual ocular hypotensive effect of Horner's syndrome. This possibility seems to be the most satisfactory explanation.

A long follow-up of patients with FHC and Horner's syndrome illustrates the therapeutic difficulties encountered when this peculiar combination is complicated by glaucoma. The reason for the complete resistance of ocular hypertension to medical and surgical therapy in such cases is not clear.

Additional studies are necessary to evaluate the 
incidence of sympathetic nervous damage in patients with FHC and to perceive its effect on the outcome of the glaucoma.

\section{References}

1 Fuchs E. Ueber Komplikationen der Heterochromie. Z Augenheilkd 1906; 15: 191-212.

2 Franceschetti A. Heterochromic cyclitis (Fuchs'syndrome). Am J Ophthalmol 1955; 39: 50-8.

3 Huber A. Das Glaucom bei komplizierten Heterochromie Fuchs. Ophthalmologica 1961; 142: 66-115.

4 Kimura SJ, Hogan MJ, Thygeson P. Fuchs' syndrome of heterochromic cyclitis. Arch Ophthalmol 1955; 54: 179-86.

5 Julien RG, Vincendeau J. Heterochromie de Fuchs, glaucome et sympathectomimetiques. Bull Soc Ophthalmol Fr 1956: 187-8.

6 Dernouchamps JP. Fuchs' Heterochromic cyclitis. Acta Ophthalmol (Kbh) 1984; 163 (suppl): 49.

7 Lemke L. Das Glaucoma bei Heterochromiecyclitis. Ophthalmologica 1966; 151: 457-64.

8 Liesegang TJ. Clinical features and prognosis in Fuchs' uveitis syndrome. Arch Ophthalmol 1982; 100: 1622-6.

9 O'Connor GR. Heterochromic iridocyclitis. Trans Ophthalmol Soc UK 1968; 104: 219-30.

10 O'Connor GR. Heterocromic iridocyclitis. Doyne lecture. Trans Ophthalmol Soc UK 1985; 104: 219-31.

11 de Abreu MT, Belfort R, Hirata P. Fuchs' heterochromic cyclitis and ocular toxoplasmosis. Am J Ophthalmol 1982; 93: 739-44.

12 Arffa RC, Schlaegel Jr TF. Chorioretinal scars in Fuchs' heterochromic iridocyclitis. Arch Ophthalmol 1984; 102: 1153-5.

13 Passow A. Hornersyndrom, Heterochromic, und Status dystrophicus, ein Symptomkomplex. Arch Augenheilkd 1933; 197: $1-51$.

14 Calmettes ML, Deodati R, Amalric P. Un cas d'association d'heterochromic de Fuchs et de syndrome de Claude BernardHorner. Rev Oto-Neuro-Ophthalmol 1953; 25: 399-400).

15 Smith RE, O'Connor GR. Cataract extraction in Fuchs' syndrome. Arch Ophthalmol 1974; 91: 39-41.

16 Ward DM, Hart CT. Complicated cataract extraction in Fuchs heterochromic uveitis. BrJ Ophthalmol 1967; 51: 530-8.

17 Norn MS. Cataract extraction in Fuchs' heterochromia. Acta Ophthalmol (Kbh) 1968; 46: 685-99.

18 Hart CT, Ward DM. Intraocular pressure in Fuchs' heterochromic uveitis. Br J Ophthalmol 1967; 51: 739-43.

19 Chandler PA, Grant WM. Lectures on glaucoma. Philadelphia: Lea and Febiger, 1965.

20 Velicky J. Vyskyt a diagnostika glaucomu pri komplikovane heterocrommi. Cesk Oftalmol 1964; 20: 298-301.

21 Sears ML, Barany EH. Outflow resistance and adrenergic mechanism. Arch Ophthalmol 1960; 64: 839-48.

22 Laugham ME, Taylor $\mathrm{CB}$. The influence of pre- and postganglionic section of the cervical sympathetic on the intraocular pressure of rabbits and cats. J Physiol (Lond) 1960; 152: 437-46.

23 Abadie C. Nature et traitement du glaucome. Arch Ophtalmol (Paris) 1899; 19: 94-101.

24 Ionesco T. Die Resection des Halssympaticus in der Behandlung des Glaukoma. Wien Klin Wochenschr 1899; 12: 483-6.

25 Swegmark G. Aqueous humour dynamics in Horner's syndrome. Trans Ophthalmol Soc UK 1963; 83: 255-61.

26 Wentworth WO, Brubanker RF. Aqueous humor dynamics in a series of patients with third neuron Horner's syndrome. Am J Ophthalmol 1981; 92: 407-15.

27 Weinstein GM, Laugham ME. Horner's syndrome and glaucoma. Arch Ophthalmol 1969; 82: 483-6.

28 Brazier DJ. Ocular sympathetic denervation associated with ocular hypertension: a case report. Br J Ophthalmol 1983; 67: 297-301.

Accepted for publication 31 October 1986. 